\title{
A B-STAR HIGH LUMINOSITY INDICATOR
}

\author{
I. Furenlid
}

Kitt Peak National Observatory

W. L. Sanders

New Mexico State University

In an effort to sort out BIa supergiants among B-stars

for use in delineating spiral structure, a photometric discriminate has been sought in the following way. Wide slit $16 \AA / \mathrm{mm}$ (flux) spectra were obtained of a sample of B stars over a suitable spectral and luminosity range. These spectra were digitized on the PDS Microdensitometer. Numerical filters of various widths were then slid along the spectra and numerous color-indices were formed and tested. An index $\ell_{0}=u+v-1.9(\ell)$ plotted against $c_{0}$, where $\ell$ is a $200 \AA$ halfwidth passband centered near $3840 \AA$ and the other magnitudes are on the Strömgren 4-color system, succeeds in separating the Ia supergiants up to Bl. For stars of spectral type Bl and earlier, confusion sets in and the separation is uncertain (although a fair percentage of success is also had for types BI and BO). This luminosity discriminate is now in use for a large spiral structure program in which all known Be stars (and perhaps eventually all B stars) are being surveyed for Ia supergiants. Spectra for 2-dimensional classification are obtained for the Ia candidates. 